\title{
Category of manuscript
}

Original article

\section{Article title}

The relationship between parenting stress and children's behavioral characteristics in Japan

\section{Author names and affiliations}

Akiko Tokunaga $^{1}$, Ryoichiro Iwanaga ${ }^{1}$, Yoko Yamanishi ${ }^{1,2}$, Takuya Higashionna ${ }^{1,3}$, Koji Tanaka ${ }^{1}$, Hideyuki Nakane ${ }^{1}$, Goro Tanaka ${ }^{1}$

${ }^{1}$ Department of Psychiatric Rehabilitation Science, Nagasaki University Graduate School of Biomedical Sciences, 1-7-1 Sakamoto, Nagasaki, Japan

${ }^{2}$ Department of Occupational Therapy, Prefectural University of Hiroshima, 1-1 Gakuenmachi, Mihara, Hiroshima, Japan

${ }^{3}$ Misakaenosono Mutsumi Developmental, Medical and Welfare Center, 570-1,Konagaicho Maki, Isahaya, Nagasaki,Japan

\section{Author contribution}

A.T.,R.I.,Y.Y.,and G.T. designed the study; A.T.,T.H.,K.T,and G.T. collected data; A.T.,R.I., Y.Y.,H.T.K.T, H.N and G.T. analyzed data and wrote the manuscript. All authors contributed to the intellectual content of this manuscript and approved the final manuscript as submitted.

\section{Finance statement}

This study did not receive any specific funding. 


\section{Conflict of interests}

The authors have no conflict of interests to declare.

Word length for the main text excluding references, abstract and tables 2747

Word length of the abstract

203

The number of references, figures and tables

23,0 and 4

Corresponding author

Akiko Tokunaga

ORCID ID 0000-0002-1508-0838

Department of Psychiatric Rehabilitation Science, Nagasaki University Graduate School of Biomedical Sciences, 1-7-1 Sakamoto, Nagasaki, Japan

Email: akiko0923@nagasaki-u.ac.jp

Tel \& Fax: +81-95-819-7996 


\begin{abstract}
Background: It has been reported that the evaluation of a child tends to differ between the mother and father regardless of whether the child has a disability or not, although parents have key information about the behavioral characteristics of the child. However, the number of reports in Japan is limited. We, therefore, investigated the relationship between the parenting stress experienced by parents of nonclinical preschool children and the children's behavioral characteristics in the present study.
\end{abstract}

Method: The subjects were 83 pairs of mothers and fathers with nonclinical children in kindergarten and nursery school (average age: $59.1 \pm 13.0$ months, 36 boys, 47 girls). The study was conducted using the Parenting Stress Index-Short Form (PSI-SF) and the Strengths and Difficulties Questionnaire (SDQ).

Results: There was no difference in the PSI-SF scores between mothers and fathers, but hyperactivity/inattention, peer relationship problems, and the total score of the SDQ were significantly higher in fathers. The results of a multiple regression analysis showed that parenting stress experienced by fathers was significantly related to hyperactivity/inattention, while parenting stress experienced by mothers was significantly related to peer relationship problems, prosocial behavior, and emotional symptoms.

Conclusion: Results suggest that children's behavioral characteristics related to parenting stress differ between mothers and fathers. 
PARENTING STRESS AND CHILDREN'S BEHAVIOR

\section{Keywords}

Parent report, Parenting stress, Child behavior, Preschoolers, Mothers and Fathers

\section{Key Notes}

- Parenting stress experienced by fathers was significantly related to hyperactivity/inattention.

- Parenting stress experienced by mothers was significantly related to peer relationship problems, prosocial behavior, and emotional symptoms.

- Results suggest that children's behavioral characteristics related to parenting stress differ between mothers and fathers.

\section{Background}

In recent years, supporting children with Autism Spectrum Disorder (ASD) or Attention Deficit Hyperactivity Disorder (ADHD) and their parents has become an important role for pediatricians and therapists ${ }^{1,2}$. Evaluation of parenting stress experienced by each parent as well as evaluation of the parents' understanding of the behavioral characteristics of the child is highly necessary when supporting the parents ${ }^{3-5}$.

There are a number of reports on the relationship between parenting stress and behavioral characteristics of children ${ }^{6-10}$. Puff et al ${ }^{6}{ }^{6}$ studied 124 parents with children aged 2 to 6 years in the United States, and reported that there was a significant relationship between 
parenting stress and the children's internalizing/externalizing problems. Buodo et al. ${ }^{7}$ studied 61 mothers with children aged 9 to 12 years in Italy, and reported that there was a significant relationship between parenting stress and the children's externalizing behavior. Saisto et al. ${ }^{8}$ studied 214 pairs of mothers and fathers with children aged 2 to 3 in Finland, and reported the child's temperament as a predictive factor of parenting stress. Ostberg et al. ${ }^{9}$ studied 1081 mothers with children aged 6 months to 3 years in Sweden, and reported behavioral problems (child fussiness-difficultness and child irregularity) of the child as predictive factors of parenting stress. Donenberg et al. ${ }^{10}$ studied 64 pairs of mothers and fathers with children aged 3.5 to 6 years in the United States, and reported that there was a significant relationship between parenting stress and the children's externalizing behavior.

However, it has been reported that the evaluation of a child tends to differ between the mother and father regardless of whether the child has a disability or not, although parents have key information about the child's behavioral characteristics ${ }^{3,11,12}$. Van der Veen-Mulders et al. ${ }^{3}$ studied the parents of 72 preschool children with ADHD (average age: 54.8 months) and 80 preschool children with no ADHD (average age: 47.3 months) in the Netherlands, and reported that the mothers of children with ADHD evaluated their children's externalizing behavior problems severely compared to the fathers and that this discrepancy between the parents was related to differences in parenting stress between the parents. Chiorri et al. ${ }^{11}$ studied 695 pairs of mothers and fathers with children (average age: 4.3 years) in the United Kingdom, and 
reported that, compared to the mothers, the fathers evaluated their children's hyperactivity/inattention, conduct problems and emotional symptoms highly while they evaluated prosocial behavior as low. Davé et al. ${ }^{12}$ studied 248 pairs of mothers and fathers with children aged 4 to 6 years in the United Kingdom, and reported that, compared to the mothers, the fathers evaluated their children's externalizing behavior highly and that the more time the fathers spent with their children, the more highly they evaluated their children's hyperactivity/inattention.

On the other hand, Amrock et al. ${ }^{13}$ conducted a large-scale survey of 21,314 parents with children aged 4 to 17 years in the United States, and reported that, regardless of the gender of the parents, there was a negative relationship between the parents' psychological distress and the children's mental health.

As described above, there are various research reports on parenting stress and children's behavioral characteristics, and differences due to the gender of the patients and children (e.g. Davé et al. ${ }^{12}$ ) have also been reported. However, the number of studies on parenting stress with nonclinical Japanese children as the subjects is limited ${ }^{14,15}$, and further research is required.

Therefore, the present study was conducted with the objective of clarifying the relationship between parenting stress experienced by parents of nonclinical Japanese preschool children and the children's behavioral characteristics. 


\section{Methods}

The design of this research is a cross-sectional study using an anonymous self-completed questionnaire survey. The present study was approved by the Ethics Review Committee of Nagasaki University Graduate School of Biomedical Sciences (approval number 13072536).

\section{Participants and procedure}

The subjects were 485 mothers and fathers with nonclinical preschool children in three kindergartens and three nursery schools in Nagasaki city. The survey period was between December 2013 and May 2014. We obtained the approval of the facility director of each facility prior to conducting the survey. An anonymous self-completed questionnaire was used for the survey. The questionnaire forms were sent to the facilities by the researchers, and the staff of each facility distributed them to the subjects. The subjects submitted the sealed envelopes containing the questionnaire form to each facility, only when they agreed to participate in this survey. Subsequently, the researchers collected what was compiled by each facility.

\section{Measures}

PSI-SF (Parenting Stress Index-Short Form)

The PSI Short Form (PSI-SF) was used to measure parenting stress. This is a short version of the Parenting Stress Index (PSI) developed by Abidin ${ }^{16}$. The PSI-SF is derived from the 101 items of the original PSI and consists of 36 items in total. The PSI-SF comprises three subscales, 
"Parental Distress: PD," "Parent-Child Dysfunctional Interaction: PCDI," and "Difficult Child: DC." The response is selected from "Strongly Agree" (5 points), "Agree" (4 points), "Not Sure" (3 points), "Disagree" ( 2 points), and "Strongly Disagree" (1 point). Higher scores indicate greater parenting stress.

SDQ (Strengths and Difficulties Questionnaire)

The SDQ developed by Goodman ${ }^{17}$ was used for the behavioral characteristics of the children. The SDQ consists of 25 items under 5 subscales of "conduct problems," "hyperactivity/inattention," "emotional symptoms," "peer problems," and "prosocial behavior." Each item is evaluated on a three-point scale of "true" ( 2 points), "somewhat true" (1 point), and "not true" (0 points). The TDS (Total Difficulties Score) is calculated based on the total score of subscales excluding "prosocial behavior" to determine the overall necessity for support.

Higher scores indicate that the needs for support are greater.

\section{Statistical analysis}

Differences in the PSI-SF and SDQ scores between the mothers and fathers were analyzed using t-tests. Multiple regression analysis was performed with the PSI-SF as a dependent variable and the children's gender, age, and the SDQ as independent variables to examine the relationship between the PSI-SF and SDQ. The data were analysed with SPSS Statistics software, version 22.0 (SPSS Inc, Chicago, IL, USA). Significance levels were set at $\mathrm{P}<0.05$. 


\section{Results}

We received responses from 122 fathers (response rate: 25.2\%) and 204 mothers (response rate: $42.1 \%)$. We analyzed the responses of 83 pairs of mothers and fathers after excluding responses with missing values or those that responded that their children had a disability.

Table 1 shows the basic attributes of the subjects. The average age of the children whose behavioral characteristics were assessed was $59.1 \pm 13.0$ months (range: 25 to 79 ). There were 36 boys (43.4\%) and 47 girls (56.6\%). There were 47 first children (56.6\%), 28 second children $(33.7 \%)$, and 8 third children $(9.6 \%)$. The average age of the fathers was $37.9 \pm 6.3$ years (range: 25 to 59 ) and the average age of the mothers was $35.1 \pm 4.9$ years (range: 24 to 46).

Table 2 shows the results of a comparison of the total scores and subscale scores of the PSI-SF between the mothers and fathers as well as between the genders of the children. PCDI, DC, and the total scores of the PSI-SF as assessed by the mothers tended to be higher $(p<0.1)$ in boys than those assessed by the fathers, but there were no significant differences.

Table 3 shows the results of a comparison of the TDS and subscale scores of the SDQ between the mothers and fathers as well as between the genders of the children. The TDS $(\mathrm{p}<0.05, \mathrm{~d}=0.36)$ and subscales of hyperactivity/inattention $(\mathrm{p}<0.01, \mathrm{~d}=0.51)$ and peer problems $(p<0.05, d=0.43)$ were assessed as significantly higher by fathers than mothers. Additionally, with regard to the gender of the children, fathers evaluated the TDS $(p<0.01, d=$ 
$0.59)$, and the subscale scores of hyperactivity/inattention $(\mathrm{p}<0.01, \mathrm{~d}=0.71)$ and peer problems $(\mathrm{p}<0.05, \mathrm{~d}=0.50)$ significantly higher in girls than mothers.

Table 4 shows the results of multiple regression analyses performed separately for mothers and fathers with the PSI-SF as a dependent variable and the children's gender/age and SDQ as independent variables. The total score of the PSI-SF was significantly related to hyperactivity/inattention ( $(=0.377, \mathrm{p}<0.01)$ in fathers, while it was significantly related to emotional symptoms $(\beta=0.216, \mathrm{p}<0.05)$, peer problems $(\beta=0.263, \mathrm{p}<0.01)$, and prosocial behavior $(\beta=-0.224, p<0.05)$ in mothers. With regard to the subscales of the PSI-SF, PD and emotional symptoms, PCDI and the gender of the child $(\beta=-0.228, \mathrm{p}<0.05)$ as well as hyperactivity/inattention $(\beta=0.413, \mathrm{p}<0.01)$, and $\mathrm{DC}$ and conduct problems $(\beta=0.378$, $\mathrm{p}<0.01)$ as well as hyperactivity/inattention $(\beta=0.341, \mathrm{p}<0.01)$ were significantly related in the fathers. In the mothers, PCDI and the age of the child $(\beta=0.216, p<0.05)$, peer problems $(ß=0.421, \mathrm{p}<0.01)$ as well as prosocial behavior $(\beta=-0.307, \mathrm{p}<0.01)$, and $\mathrm{DC}$ and emotional symptoms $(\beta=0.289, \mathrm{p}<0.01)$ as well as hyperactivity/inattention $(\beta=0.288, \mathrm{p}<0.05)$ were significantly related.

\section{Discussion}

The purpose of the present study was to clarify the relationship between parenting stress experienced by parents of nonclinical preschool children and the children's behavioral 
characteristics in Japan.

In the present study, PCDI, DC, and the total scores of the PSI-SF consisting of 36 items as evaluated by the mothers tended to be higher in boys than those evaluated by the fathers, but there were no significant differences. Rolle et al. ${ }^{18}$ compared the scores of the PSISF consisting of 36 items for 134 pairs of mothers and fathers with children aged 1 year (boys: 61\%, girls: $39 \%$ ), and reported that PD was higher in mothers. Van der Veen-Mulders et al. ${ }^{3}$ compared the scores of PSI-SF consisting of 25 items for parents of 72 preschool children with ADHD (average age: $54.8 \pm 11.0$ months; boys: $81 \%$, girls: $19 \%$ ) and 80 preschool children with no ADHD (average age: $47.3 \pm 12.7$ months; boys: 53\%, girls: $47 \%$ ), and reported that the total PSI score was higher in mothers of children with ADHD than fathers, while there was no significant difference between the mothers and fathers of children with no ADHD. Davis et al. ${ }^{4}$ compared the scores of PSI-SF consisting of 36 items for 54 couples of mothers and fathers of children with ASD (average age: $26.9 \pm 4.2$ months; boys: $74 \%$, girls: $26 \%$ ) in the United States, and reported that DC and PD were higher in mothers. Yeh et al. ${ }^{19}$ compared the scores of PSI-SF consisting of 15 items for 100 mothers and 49 fathers of children with cancer under the age of 18 years (median age: 5.75 years, range: 0.4 to 15.8 ; boys: $69.13 \%$, girls: $30.2 \%$ ) in Taiwan, and reported that there was no significant difference between the mothers and fathers. Baker et al. ${ }^{5}$ compared the scores of the Family Impact Questionnaire (Donenberg et al. ${ }^{10}$ ), which measures parenting stress, for the parents of 82 children with delayed development 
(average age: $35.6 \pm 2.86$ months; boys: $66 \%$, girls: $34 \%$ ) and 123 children with no delayed development (average age: $34.9 \pm 3.13$ months; boys: 53\%, girls: 47\%), and reported that, regardless of whether the children suffered from delayed development or not, there was no difference between the mothers and fathers. The results of the previous studies we have seen so far show that, although there is variation in parenting stress of the parents depending on the age of the children studied and whether the children studied have a disability, the differences between the mothers and fathers of the nonclinical children are generally small, and we believe they support the results of the present study.

In the present study, the TDS, and the scores of hyperactivity/inattention and peer problems of the SDQ were higher in fathers than mothers. Additionally, fathers considered the TDS, hyperactivity/inattention, and peer problems of their female children significantly higher than mothers. D'Souza et al. ${ }^{20}$ studied 3759 fathers and 6246 mothers with children aged 2 years in New Zealand using the SDQ, and reported that emotional symptoms, hyperactivity/inattention, and the TDS were higher in fathers than mothers, while prosocial behavior was lower. Chiorri et al. ${ }^{11}$ studied 695 pairs of mothers and fathers with children (average age: 4.25 year) in the United Kingdom using the SDQ, and reported that fathers evaluated hyperactivity/inattention, conduct problems, and emotional symptoms higher than mothers, while fathers evaluated prosocial behavior lower than mothers. Griffith et al. ${ }^{21}$ studied 130 fathers and 168 mothers of 168 siblings with ASD (average age: $10.5 \pm 3.4$ years; 85 boys, 
83 girls) using the SDQ, and reported that mothers evaluated prosocial behavior higher than fathers. Mellor et al. ${ }^{22}$ studied the mothers and fathers of 700 elementary school children (average age: $8.7 \pm 1.69$ years; 320 boys, 380 girls) in China, and reported that mothers evaluated prosocial behavior higher than fathers. Davé et al. ${ }^{12}$ studied 248 dyads of mothers and fathers with children aged 4 to 6 years (boys: 53\%; girls: 47\%) in the United Kingdom using the SDQ, and reported that fathers evaluated hyperactivity/inattention, conduct problems, and the TDS higher than mothers, while conduct problems and the TDS were higher in female children. Furthermore, they reported that the longer the time a father spent with his child, the higher they evaluated hyperactivity/inattention. Comparison of the results of the abovementioned previous studies and the present study suggests that fathers tend to be more sensitive to externalizing behaviors than mothers or tend to evaluate them excessively highly. Moreover, this tendency is believed to be affected by the gender and age of the children studied, and time spent with the children. On the other hand, the fact that children show different behaviors to the mother and father, respectively, may also need to be considered ${ }^{3,12}$.

In the present study, multiple regression analyses were performed separately for mothers and fathers with the PSI-SF as a dependent variable, and the gender/age of the children and the SDQ as independent variables. The results showed that the total PSI-SF score was significantly related to hyperactivity/inattention of the SDQ in fathers, while it was significantly related to peer problems, prosocial behavior, and emotional symptoms in mothers. 
Davis et al. ${ }^{4}$ studied 54 parents of children with ASD in the United States. The results of a multiple regression analysis showed that regulatory problems (e.g., eating, sleeping, and emotion regulation) were related to maternal stress, while externalizing behaviors were related to paternal stress. Beck et al. ${ }^{23}$ studied 74 mothers of children with intellectual disabilities (average age: 9.8 years), and reported that the more problematic behavior and less prosocial behavior there was, the greater stress they experienced, and that high stress levels of mothers could be predicted based on the children's lack of prosocial behavior. Ostberg et al. ${ }^{9}$ studied 1081 mothers with children aged 6 months to 3 years in Sweden, and reported problematic behaviors of the children (child fussiness-difficultness and child irregularity) as a predictive factor for parenting stress. In summary, regardless of whether the child has a disability or not, parenting stress experienced by fathers can be observed objectively but is difficult to manage, and is thus related to externalizing behaviors, which tend to characteristically receive a negative evaluation in social settings. On the other hand, it was suggested that parenting stress experienced by mothers was related to concerns for interpersonal relationships with the people around them such as peer problems, and for the children's internal aspects such as emotional symptoms. Therefore, we believe it is necessary to evaluate different forms of support for mothers and fathers when providing parenting support to them.

\section{Study Limitations}

In the present study, basic attributes such as educational background and parents' occupation, 
mental health, marital relationship, alcohol-drinking history, and time spent with children were not included in the survey items. The relationship between these attributes and parenting stress remains to be studied. The sample size of the present study is small compared to previous studies, and the generalizability of the results is limited. Going forward, we seek to increase the sample size and study parents of children with ASD and ADHD.

\section{References}

1 Barnes G, Wilkes-Gillan S, Bundy A, \& Cordier R. The social play, social skills and parent-child relationships of children with ADHD 12 months following a RCT of a playbased intervention. Australian Occupational Therapy Journal, 2017; 64, 457-465.

2 Cantrill A, Wilkes-Gillan S, Bundy A, Cordier R, \& Wilson NJ. An eighteen-month follow-up of a pilot parent-delivered play-based intervention to improve the social play skills of children with attention deficit hyperactivity disorder and their playmates. Australian Occupational Therapy Journal, 2015; 62(3),197-207.

3 van der Veen-Mulders L, Nauta MH, Timmerman ME, van den Hoofdakker BJ, Hoekstra PJ. Predictors of discrepancies between fathers and mothers in rating behaviors of preschool children with and without ADHD. European Child \& Adolescent Psychiatry,2017; 26(3), 365-376.

4 Davis NO, \& Carter AS. Parenting Stress in Mothers and Fathers of Toddlers with Autism 
Spectrum Disorders: Associations with Child Characteristics. Journal of Autism and Developmental Disorders, 2008; 38, 1278-1291.

5 Baker BL, McIntyre LL, Blacher J, Crnic K, Edelbrock C, \& Low C. Pre-school children with and without developmental delay: behaviour problems and parenting stress over time. Journal of Intellectual Disability Research, 2003; 47, 217-230.

6 Puff J \& Renk K. Relationships among parents' economic stress, parenting, and young children's behavior problems. Child Psychiatry and Human Development, 2014; $45(6), 712-27$.

7 Buodo G, Moscardino U, Scrimin S, Altoe` G, \& Palomba D. Parenting Stress and Externalizing Behavior Symptoms in Children: The Impact of Emotional Reactivity. Child Psychiatry \& Human Development, 2013; 44(6),786-797.

8 Saisto T, Salmela-Aro K, Nurmi J, \& Halmesmaki E. Longitudinal study on the predictors of parental stress in mothers and fathers of toddlers. Journal of Pshchosomatic Obstetrics and Gynecology, 2008; 29(3), 213-222.

9 Östberg M \& Hagekull B. A Structural Modeling Approach to the Understanding of Parenting Stress. Journal of Clinical Child Psychology, 2000; 29(4), 615-625.

10 Donenberg G, \& Baker BL. The impact of young children with externalizing behaviors on their families. Journal of Abnormal Child Psychology, 1993; 21(2), 179-198.

11 Chiorri C, Hall J, Casely-Hayford J, \& Malmberg LE. Evaluating Measurement 
Invariance Between Parents Using the Strengths and Difficulties Questionnaire (SDQ). Assessment. 2016; 23(1), 63-74.

12 Davé S, Nazareth I, Senior R, \& Sherr L. A Comparison of Father and Mother Report of Child Behaviour on the Strengths and Difficulties Questionnaire. Child Psychiatry and Human Development. 2008; 39(4), 399-413.

13 Amrock SM, \& Weitzman M. Parental Psychological Distress and Children's Mental Health: Results of a National Survey. Academic Pediatrics, 2014; 14, 375-381.

14 Suzuki S. The effects of marital support, social network support, and parenting stress on parenting: self-efficacy among mothers of young children in Japan. Journal of Early Childhood Research, 2010; 8(1), 40-66.

15 Suzuki S, Holloway SD, Yamamoto Y, \& Mindnich JD. Parenting self-efficacy and social support in Japan and United States. Journal of Family Issues, 2009; 30, 1505-1526,

16 Abidin RR. Parenting Stress Index, Third Edition: Professional Manual. Odessa, FL: Psychological Assessment Resources, Inc.,1995.

17 Goodman R. The Strengths and Difficulties Questionnaire: A Research Note. The Journal of Child Psychology and Psychiatry, 1997; 38(5), 581-586.

18 Rolle L, Prino LE, Sechi C, Vismara L, Neri E, Polizzi C, Trovato A, Volpi B, Molgora S, Fenaroli V, Ierardi E, Ferro V, Lucarelli L, Agostini F, Tambelli R, Saita E, Riva Crugnola C, Brustia P. Parenting Stress, Mental Health, Dyadic Adjustment: A Structural Equation 
Model. Frontiers in Psychology, 2017; 8, 839-839.

19 Yeh $\mathrm{CH}$, Chen ML, Li W, \& Chuang HL. The Chinese version of the Parenting Stress Index: a psychometric study. Acta paediatrica,2001; 90,1470-1477.

20 D'Souza S, Waldie KE, Peterson ER, Underwood L, \& Morton SMB. The Strengths and Difficulties Questionnaire: Factor Structure of the Father-Report and Parent Agreement in 2-Year-Old Children. Assessment, 2017; Mar 1:1073191117698757. doi: 10.1177/1073191117698757. [Epub ahead of print]

21 Griffith GM, Hastings RP, \& Petalas MA. Brief Report: Fathers' and Mothers' Ratings of Behavioral and Emotional Problems in Siblings of Children with Autism Spectrum Disorder. Journal of Autism and Developmental Disorders, 2014; 44(5), 1230-1235.

22 Mellor D, Wong J, \& Xiaoyan X. Interparent Agreement on the Strengths and Difficulties Questionnaire: A Chinese Study. Journal of Clinical Child \& Adolescent Psychology,2011; 40(6), 890-896.

23 Beck A, Hastings RP, Daley D, \& Stevenson J. Prosocial behaviour and behaviour problems independently predict maternal stress. Journal of Intellectual and Developmental Disability, 2004; 29(4), 339-349. 
Table 1 Sample characteristics

\begin{tabular}{lrr} 
& & $\mathrm{n}(\%)$ \\
\hline Sex & & \\
& Boys & $36(43.4)$ \\
& Girls & $47(56.6)$ \\
Birth order & & \\
& $1 \mathrm{st}$ & $47(56.6)$ \\
& 2nd & $28(33.7)$ \\
& $3 \mathrm{rd}$ & $8(9.6)$ \\
& & \\
& & Mean (SD) \\
\hline Child's age (months) & & $59.1(13.0)$ \\
Mother's age (years) & & $35.1(4.9)$ \\
Father's age (years) & & $37.9(6.3)$ \\
\hline
\end{tabular}


Table 2 Mean differences between fathers' and mothers' PSI-SF scores

\begin{tabular}{llrrrl}
\hline PSI-SF & & \multicolumn{1}{c}{ Fathers } & Mothers & & $\begin{array}{l}\text { Effect } \\
\text { size }\end{array}$ \\
\hline PD & All & $25.7(5.3)$ & $27.2(6.3)$ & 0.101 & 0.26 \\
& Boys & $26.0(5.6)$ & $27.2(6.6)$ & 0.410 & 0.20 \\
& Girls & $25.4(5.2)$ & $27.1(6.1)$ & 0.144 & 0.30 \\
\hline PCDI & All & $19.4(5.2)$ & $20.3(5.2)$ & 0.252 & 0.17 \\
& Boys & $18.7(5.5)$ & $21.0(5.7)$ & 0.084 & 0.41 \\
& Girls & $20.0(4.8)$ & $19.8(4.9)$ & 0.899 & 0.04 \\
\hline DC & All & $25.7(7.0)$ & $27.1(7.0)$ & 0.194 & 0.20 \\
& Boys & $25.3(7.6)$ & $28.8(7.8)$ & 0.063 & 0.46 \\
& Girls & $26.0(6.7)$ & $25.9(6.2)$ & 0.936 & 0.02 \\
\hline Total & All & $70.7(14.1)$ & $74.6(15.3)$ & 0.095 & 0.27 \\
& Boys & $70.0(15.4)$ & $76.9(16.9)$ & 0.074 & 0.43 \\
& Girls & $71.3(13.3)$ & $72.8(13.9)$ & 0.597 & 0.11 \\
\hline
\end{tabular}

PSI-SF, Parenting Stress Index-Short Form; PD, Parental Distress;

PCDI, Parent-Child Dysfunctional Interaction; DC, Difficult Child

SD, standard deviation; Effect size, Cohen's $d$

$\mathrm{t}$ test $* \mathrm{p}<0.05 * * \mathrm{p}<0.01$ 
Table 3 Mean differences between fathers' and mothers' SDQ scores

\begin{tabular}{|c|c|c|c|c|c|}
\hline SDQ & & $\begin{array}{c}\text { Fathers } \\
\text { Mean (SD) }\end{array}$ & $\begin{array}{c}\text { Mothers } \\
\text { Mean (SD) }\end{array}$ & $\mathrm{p}$ & $\begin{array}{l}\text { Effect } \\
\text { size }\end{array}$ \\
\hline \multirow[t]{3}{*}{ Emotional Symptoms } & All & $2.1(1.7)$ & $1.9(1.7)$ & 0.307 & 0.12 \\
\hline & Boys & $2.0(1.4)$ & $2.1(1.9)$ & 0.889 & 0.06 \\
\hline & Girls & $2.2(1.9)$ & $1.7(1.4)$ & 0.139 & 0.30 \\
\hline \multirow[t]{3}{*}{ Conduct Problems } & All & $2.7(1.6)$ & $2.8(1.9)$ & 0.896 & 0.06 \\
\hline & Boys & $3.1(1.7)$ & $3.2(2.1)$ & 0.805 & 0.05 \\
\hline & Girls & $2.4(1.5)$ & $2.4(1.7)$ & 0.950 & 0.00 \\
\hline \multirow[t]{3}{*}{ Hyperactivity/Inattention } & All & $4.0(2.3)$ & $2.9(2.0)$ & $0.001^{* *}$ & 0.51 \\
\hline & Boys & $4.5(2.4)$ & $3.8(2.3)$ & 0.196 & 0.30 \\
\hline & Girls & $3.6(2.1)$ & $2.3(1.5)$ & $0.001^{* *}$ & 0.71 \\
\hline \multirow[t]{3}{*}{ Peer Problems } & All & $2.2(1.4)$ & $1.6(1.4)$ & $0.019^{*}$ & 0.43 \\
\hline & Boys & $2.1(1.5)$ & $1.8(1.5)$ & 0.346 & 0.20 \\
\hline & Girls & $2.2(1.4)$ & $1.5(1.4)$ & $0.022^{*}$ & 0.50 \\
\hline \multirow[t]{3}{*}{ Prosocial Behaviour } & All & $6.1(2.0)$ & $6.3(2.1)$ & 0.514 & 0.10 \\
\hline & Boys & $5.3(1.8)$ & $5.9(2.2)$ & 0.225 & 0.30 \\
\hline & Girls & $6.8(1.8)$ & $6.7(2.0)$ & 0.827 & 0.05 \\
\hline \multirow[t]{3}{*}{ Total Difficulties } & All & $11.0(4.8)$ & $9.2(5.1)$ & $0.017^{*}$ & 0.36 \\
\hline & Boys & $11.7(4.7)$ & $10.8(6.0)$ & 0.484 & 0.17 \\
\hline & Girls & $10.5(4.9)$ & $7.9(3.9)$ & $0.006^{* *}$ & 0.59 \\
\hline
\end{tabular}

SDQ, Strengths and Difficulties Questionnaire

$\mathrm{SD}$, standard deviation; Effect size, Cohen's $d$

t test $* p<0.05 * * p<0.01$ 


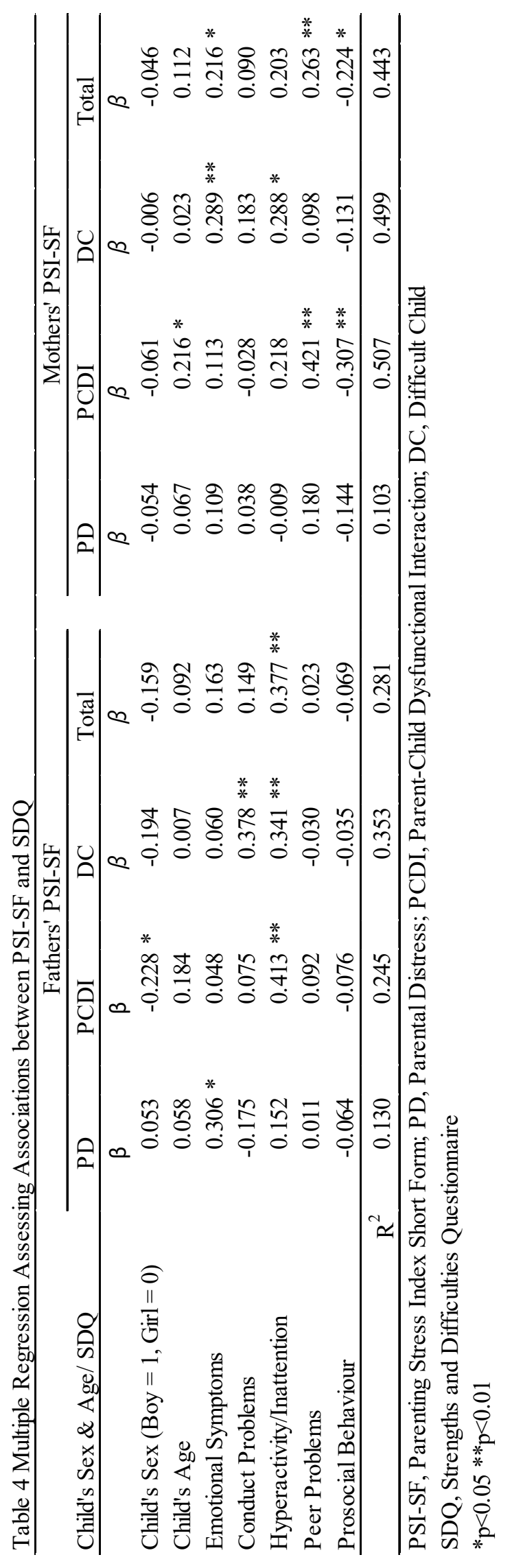

